\title{
Single Series Peptide Fragment Ion Spectra Generated by Two-Stage Collision-Induced Dissociation in a Triple Quadrupole
}

\author{
Wolf D. Lehmann \\ Central Spectroscopy Department, German Cancer Research Center, Im Neuenheimer Feld, \\ Heidelberg, Germany
}

\begin{abstract}
By using nanoelectrospray ionization and a triple quadrupole analyzer, simplified fragment ion spectra of peptides have been recorded by combining skimmer collision-induced dissociation with precursor ion scanning or neutral loss scanning. These pseudo-MS ${ }^{3}$ scan modes are characterized by two-stage collision-induced dissociation and have been termed SCID/ precursor and sCID/neutral loss scan, respectively. By these scan modes, peptide fragment ion spectra can be generated that predominantly show signals of a single fragment ion series, such as the B or $\mathrm{Y}^{\prime \prime}$ series. Skimmer collision-induced dissociation combined with scanning for neutral loss of 28 generates spectra showing $B$ ions, whereas combination with precursor ion scanning for the $Y_{1}^{\prime \prime}$ ion results in spectra showing $Y^{\prime \prime}$ ions for tryptic peptides $\left(Y_{1}^{\prime \prime}=m / z 147\right.$ for C-terminal lysine, $Y_{1}^{\prime \prime}=m / z 175$ for C-terminal arginine). Sequence information including the direction of the sequence is easily extracted from the simplified fragment ion spectra generated by two-stage collision-induced dissociation, because the scan mode defines the type of fragments observed. The analytical results reported are similar to those that have been achieved in $\mathrm{MS}^{3}$ experiments using a hybrid BEQQ or a pentaquadrupole mass spectrometer (Schey, K. L.; Schwartz, J. C.; Cooks, R. G. Rapid Commun. Mass Spectrom. 1989, 3, 305-309). The pseudo-MS ${ }^{3}$ technique used in this study has some limitations with respect to sample purity, because there is no step of mass selection before the first stage of collisional activation; however, it has the advantage that a standard triple quadrupole instrumentation can be used. (J Am Soc Mass Spectrom 1998, 9, 606-611) C C 1998 American Society for Mass Spectrometry
\end{abstract}

$\mathrm{U}$ nder the conditions of collision-induced dissociation (CID) peptide molecular ions dissociate at different sites of the peptide backbone [1-3]. This effect results in various types of fragment ions [4]. Sequence information is provided by the mass differences between adjacent ions of a single fragment ion series. However, a peptide product ion spectrum generally consists of several fragment ion series superimposed on each other, a situation that seriously hampers the extraction of sequence information. Several strategies have been introduced for the identification of fragment ion series, in particular for discrimination between $\mathrm{N}$-terminal and C-terminal fragment ions. For this purpose, e.g., selective derivatization of the $\mathrm{N}$ terminal amino function by a quaternary reagent [5], analyzing the stability of fragment ions [6], esterification of the C-terminal carboxyl function, selective labeling of the C-terminus by protease-catalyzed incorporation of oxygen-18 [7-9], or MS ${ }^{3}$ experiments involving reaction intermediate scanning [10] have been used.

Address reprint requests to Dr. Wolf D. Lehmann, Central Spectroscopy Department, German Cancer Research Center, Im Neuenheimer Feld 280, D-69120 Heidelberg, Germany. E-mail: wolf.lehmann@dkfz-heidelberg.de Presented in part at the 45th ASMS Conference on Mass Spectrometry and Allied Topics, June 1-5, 1997, Palm Springs, CA.
In the past, MS/MS analyses involving more than one step of collisional activation have been performed with hybrid instruments [10,11], pentaquadrupole instruments [10, 12], quadrupole ion trap analyzers [13, $14]$, or magnetic ion traps $[15,16]$. In the following study, it is shown that two-stage CID performed in a triple quadrupole instrument (pseudo-MS ${ }^{3}$ ) can generate simplified fragment ion spectra of peptides that are characterized by a single fragment ion series.

\section{Experimental}

Electrospray ionization (ESI) mass spectra were recorded on a TSQ 7000 triple quadrupole instrument (Finnigan, San Jose, CA) equipped with a nanoESI source (EMBL, Heidelberg, Germany). Solutions were sprayed with a needle potential of $+0.7 \mathrm{kV}$ with an estimated flow rate of $\sim 20-40 \mathrm{~nL} / \mathrm{min}$. MS/MS analyses were performed with argon as collision gas at a pressure of 2 mtorr. All spectra were acquired in profile mode by averaging about 100 consecutive scans of 4-s duration. The concentrations of peptide solutions were in the range of $1-10 \mathrm{pmol} / \mu \mathrm{L}$ in water/acetonitrile 1:1 containing $1 \%$ acetic acid. Angiotensin I was obtained 


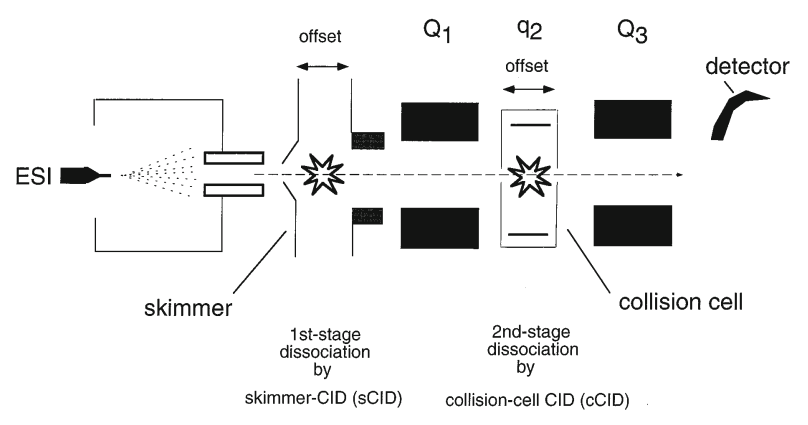

Figure 1. Location of skimmer-CID (sCID) and collision cell-CID (cCID) in a triple quadrupole analyzer combined with electrospray ionization; sCID is performed on a non-mass-selected ion beam, whereas in CCID a mass selected ion beam is fragmented.

from Sigma (St. Louis, MO), the other peptides were synthesized in-house.

\section{Results and Discussion}

\section{Basic Principle}

In a triple quadrupole ions can be collisionally activated (i) in the skimmer region (sCID) and (ii) in the collision cell (cCID) as shown in Figure 1. Both the residual gas pressure and the offset potential determine the efficiency of the CID process. For a constant pressure the intensity of a particular fragment ion in general maximizes at a typical offset potential. At offset potentials below this optimal offset the energy transfer is not optimal, at offset values above the maximum decomposition to second generation fragments prevails. Increase in the residual gas pressure shifts the maximum of an offset plot (fragment ion intensity as a function of the offset potential) to a lower offset value. Using comparable offset potentials and standard pressure conditions (2-3 mtorr He or Ar in the collision cell), both SCID and CCID yield qualitatively similar product ion spectra of peptides. A special feature of SCID is its dependence on the ESI flow rate, because evaporating solvent contributes to the gas pressure in the skimmer region. Thus, sCID with nanoESI requires somewhat higher offset potentials than standard ESI because of its much lower flow rate (data not shown). Fragmentation conditions can be optimized for a certain fragmentation process by recording of the fragment ion intensity as a function of the offset potential.

Using ESI, MS/MS experiments are, in general, used for the structural characterization of selected molecular ions, because ESI is normally not accompanied by fragmentation. By the addition of sCID, the fragmentation step in the collision cell can be used for generation of second generation fragment ions, which then provide structural information on the first generation fragment ions generated by sCID. This pseudo-MS ${ }^{3}$ approach sacrifices the exact precursor/product relationship provided by true $\mathrm{MS}^{3}$ experiments, but nevertheless may provide improved analytical information, e.g., in pep- tide sequencing, as will be demonstrated and discussed below.

\section{Generation of a Selective $Y^{\prime \prime}$-Series Spectrum}

Two-stage CID of the $[\mathrm{M}+\mathrm{H}]^{+}$ions of the peptide TRPPASPSPQR was performed as demonstrated in Figure 2. As a result of the first dissociation step performed by a skimmer-CID offset of $-40 \mathrm{~V}$ the product ion spectrum shown in Figure 2a is observed. This product ion spectrum shows the superposition of various fragment ions series.

The fragment ions generated by SCID were subjected to a second CID step in the collision cell, whereas the triple quadrupole analyzer was scanned in the precursor ion mode for $m / z 175$. The resulting spectrum is shown in Figure 2b. Precursor ion scanning for $m / z 175$ was selected since the investigated peptide carries a C-terminal arginine residue giving rise to a $\mathrm{Y}_{1}^{\prime \prime}$ ion at $m / z$ 175. Therefore, the sCID/precursor of 175 scan should provide a selective detection of $Y^{\prime \prime}$ ions, since only $Y^{\prime \prime}$ fragments form the $Y_{1}^{\prime \prime}$ ion as second generation fragment ion. The simplified spectrum in Figure $2 \mathrm{~b}$ shows that this assumption is valid because it contains predominantly $\mathrm{Y}^{\prime \prime}$ ions where the sequence from residues 1 to 9 can be directly read. An additional benefit from this SCID/precursor scan mode is that the direction of the sequence is also obtained, because the scan mode defines the observed ion series.

The scan modes that can be performed using a $\mathrm{MS}^{3}$ equipment (two collision cells and three mass analyzers) have been systematically outlined [11, 17]. One of these modes was the so called daughter/parent (product/precursor) scan that was later termed the reaction intermediate scan. The sCID/precursor scan used to generate the spectrum in Figure $2 b$ is analogous to this scan mode with the exception that in a triple quadrupole no precursor ion selection is possible before the sCID step. Consistent with the term "reaction intermediate scan," the ions of the $Y^{\prime \prime}$ series detected in Figure $2 \mathrm{~b}$ can be regarded as the set of intermediates occurring in the decomposition of the intact molecular ion into the $Y_{1}^{\prime \prime}$ fragment.

In this study the operating modes of the triple quadrupole analyzer are visualized by a set of pictograms [18] created to symbolize the different units of the mass analyzer and their functional state. This approach differs from an earlier attempt in which MS/MS scan modes were symbolized exclusively on the molecular level and independent of the type of mass spectrometer used [11].

\section{Variation of the Collision Cell Offset}

To obtain a sCID/precursor spectrum as given in Figure $2 b$, showing a distribution of fragment ion intensities similar to that observed after the stage of sCID, it is necessary to vary the offset potential in the second stage of fragmentation (cCID). This is necessary because first 


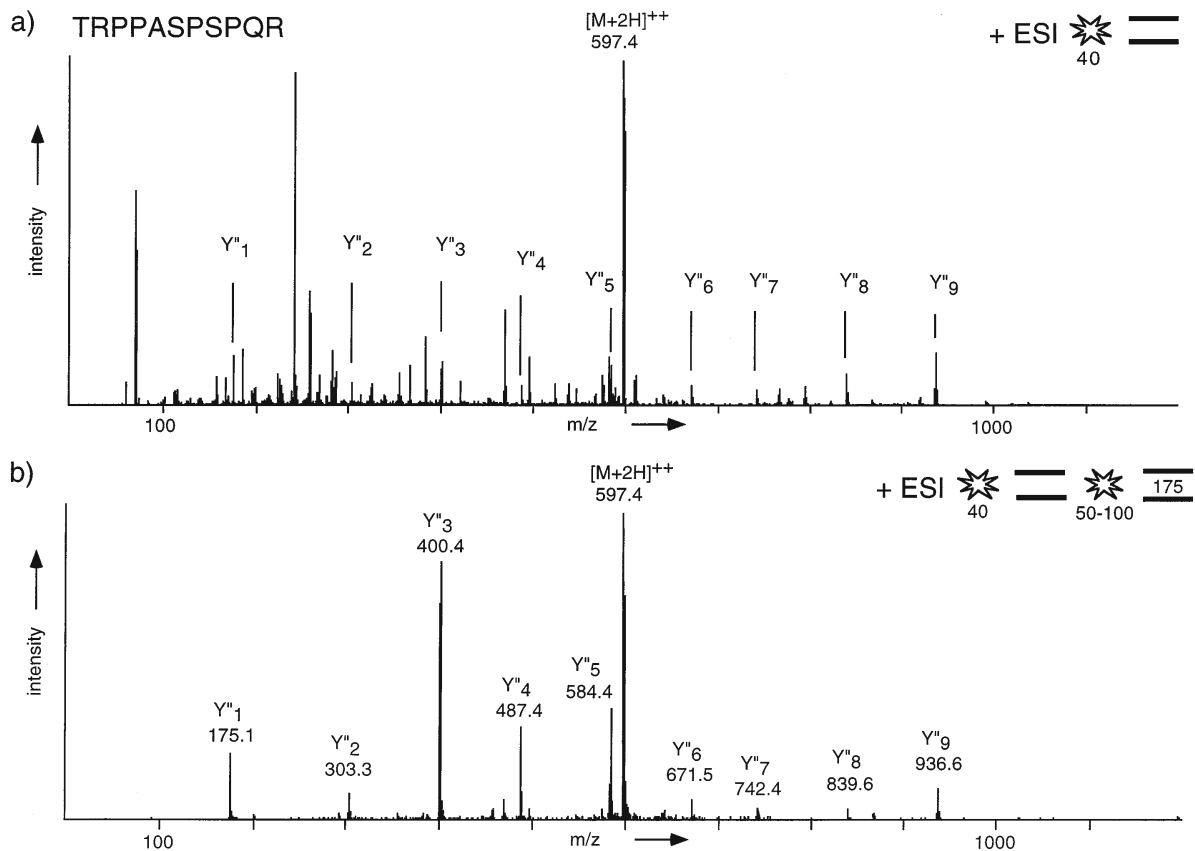

Figure 2. Positive ion nanoESI spectra of the peptide TRPPASPSPQR; (a) fragment ion spectrum generated by sCID with an offset of $-40 \mathrm{~V}$, average of 20 scans; (b) sCID/precursor of 175 scan using in addition CCID with an offset from -50 to $-100 \mathrm{~V}$, average of 100 scans. Pseudo-MS ${ }^{3}$ involving two-stage CID results in a simplified fragment spectrum showing predominantly the $\mathrm{Y}^{\prime \prime}$-ion series. The pictograms used in this study are defined in [18].

generation fragment ions of low mass have a lower optimal cCID offset for formation of $Y_{1}^{\prime \prime}$ fragments than corresponding fragments of higher mass. Variation of the cCID offset can be performed by the two techniques outlined in Figure 3. In the method called CID offset stepping (see Figure 3a) the cCID offset is increased stepwise after a preselected number of scans has been acquired (e.g., by $2 \mathrm{~V}$ every 10 scans). This method requires some time for data acquisition to cover the whole offset range of interest between $\sim 20$ and $60 \mathrm{~V}$ in addition to the SCID offset. On the other hand, this method has the advantage of not requiring previous knowledge on the fragmentation behavior of the peptide fragments. With the extremely low flow rates of nanoESI, signal averaging for improvement of the sig-
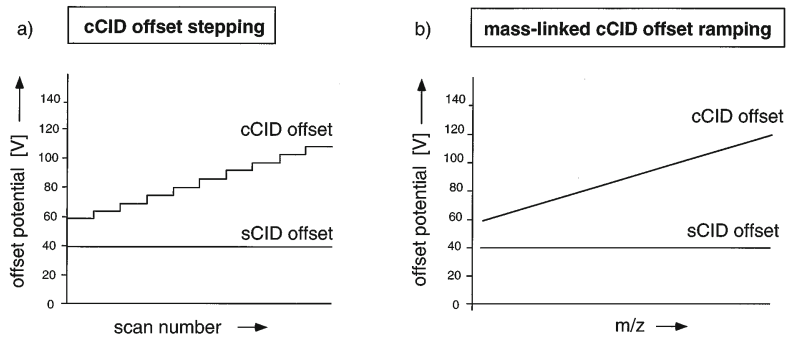

Figure 3. Schematic display of the two modes for increasing the cCID offset during acquisition of the simplified fragment ion spectra using two-stage CID; (a) cCID offset stepping with increasing scan number and (b) mass-linked cCID ramping. The spectra in this study were acquired with the offset stepping method which requires no previous knowledge of the optimal offset values. nal-to-noise ratio can be performed. If the optimal offset potentials are known, a mass-linked cCID offset ramping can be used as schematically shown in Figure 3b. In the type of instrument used, a special tune file can be created, so that the CCID offset is ramped in a preprogrammed fashion during each scan cycle. This masslinked cCID offset ramping reduces the data acquisition time required for two-stage CID analyses. The data in this study have been acquired using the offset stepping method.

\section{Generation of a Selective B-Series Spectrum}

B ions represent the most abundant type of N-terminal fragment ions of peptides. They are often accompanied by $[\mathrm{B}-28]^{+}$satellite ions, which arise from loss of $\mathrm{CO}$ from their common C-terminal structure $\left[\mathrm{R}-\mathrm{CH}\left(\mathrm{NH}_{2}\right)_{-}\right.$ CO]. This common neutral loss can be used for a selective detection of B ions by a SCID/neutral loss of 28 scan. An example is shown in Figure 4, comparing the sCID spectrum of the peptide DRVYIHPFHL in Figure $4 a$ with the sCID/neutral loss spectrum given in Figure $4 \mathrm{~b}$. As expected, the more specific spectrum in Figure $4 \mathrm{~b}$ predominantly shows $\mathrm{B}$ ions, where a part of the sequence can be read. In addition to the B-ion series, a strong signal at $m / z 110$ is found that represents the immonium ion of histidine. Upon CID, the immonium ion of histidine also shows loss of 28, which is probably due to the loss of $\mathrm{C}_{2} \mathrm{H}_{4}$. Alternatively, a selective B-ion fragment spectrum can be recorded when an abundant $B_{1}$ (or $B_{2}$ ) ion is formed using the sCID/precursor scan 


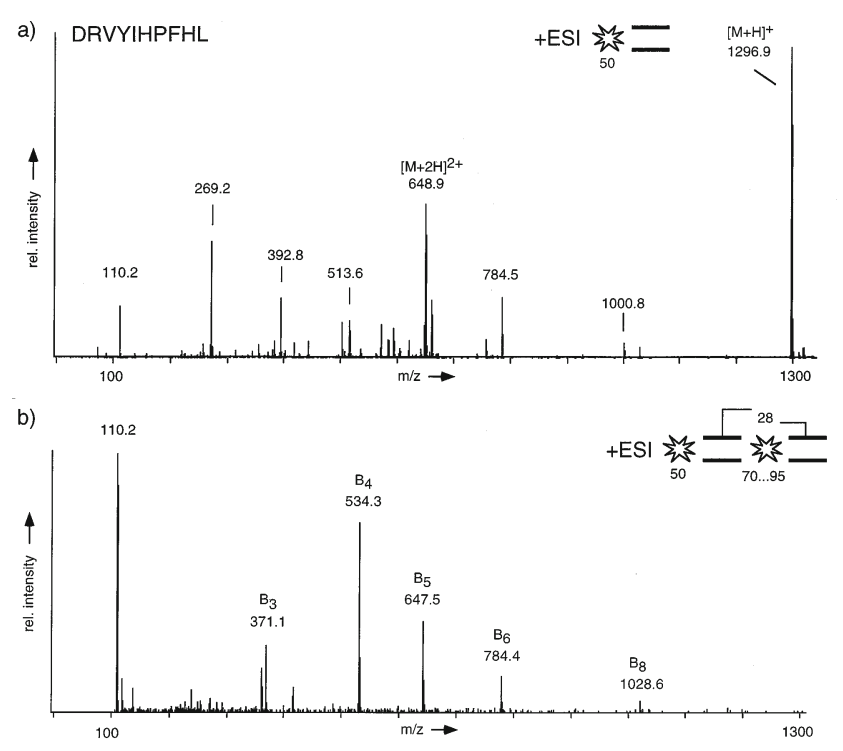

Figure 4. Positive ion nanoESI spectra of the peptide DRVYIHPFHL (angiotensin I); (a) fragment ion spectrum generated by sCID with an offset of $-50 \mathrm{~V}$, average of 20 scans; (b) sCID/ neutral loss of 28 scan using in addition cCID with an offset from -70 to $-95 \mathrm{~V}$, average of 100 scans. Pseudo-MS ${ }^{3}$ involving two-stage CID results in a simplified fragment spectrum showing predominantly the $\mathrm{B}$-ion series plus the immonium ion of histidine.

mode as applied for the generation of selective $Y^{\prime \prime}$-ion fragment spectra.

\section{Sequencing of a Modified Peptide}

Peptides carrying a covalent modification at the C- or $\mathrm{N}$-terminus that is cleaved off as a modification-specific fragment are ideally suited for sequencing by two-stage CID. This is particularly true when formation of the modification-specific fragment requires a higher offset value than is required to induce fragmentation of the peptide backbone. Such a situation is realized for myristoylated glycine that frequently occurs at the $\mathrm{N}$ terminus of the proteinkinase A family [19]. Figure 5 shows three fragment ion spectra of the peptide myrG DAAAAK which represents the deamidated T1 fragment of the catalytic subunit of bovine proteinkinase A. Figure $5 \mathrm{a}$ gives the sCID fragment ion spectrum which exhibits two superimposed fragment ion series of the $B$ and $Y^{\prime \prime}$ type, respectively. The modification-specific fragment ions for the myristoyl-glycine residue at $\mathrm{m} / \mathrm{z}$ 211,240 , and 268 are not detected at an SCID offset of $50 \mathrm{~V}$ [20]. However, their subsequent formation by cCID using an additional offset potential is an ideal probe for selective detection of the B-type fragments as demonstrated in Figure 5b. The sCID/precursor of 211 scan selectively detects the B-ion series inclusive of the intact molecular ion, which is also a direct precursor of $m / z 211$ under these conditions. The series of $Y^{\prime \prime}$ ions is selectively detected by a sCID/precursor of 147 scan

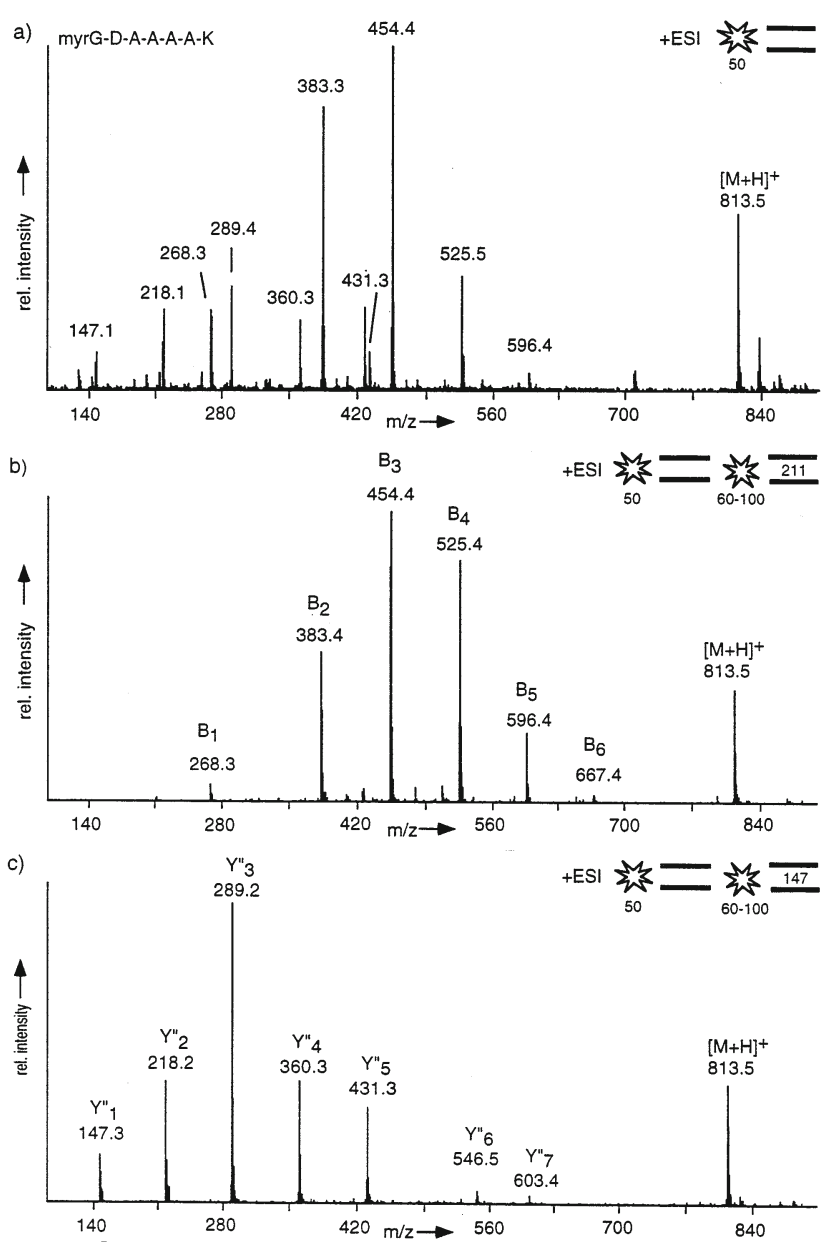

Figure 5. Positive ion nanoESI spectra of the deamidated T1 peptide the catalytic subunit of protein kinase A (bovine) myrGDAAAAK; (a) fragment ion spectrum generated by sCID $(-50 \mathrm{~V}$ offset) average of 50 scans, (b) sCID/precursor of 211 (myristoyl fragment ion) scan using in addition cCID with an offset from -60 to $-100 \mathrm{~V}$, average of 80 scans, (c) sCID/precursor of 147 ( $\mathrm{Y}_{1}^{\prime \prime}$ ion of $\mathrm{K})$ scan using the same offset conditions as in (b), average of 80 scans. The sCID/precursor scan selectively shows the B-ion series (b) or the $Y^{\prime \prime}$-ion series (c), respectively.

(Figure $5 c$ ), since this peptide exhibits a C-terminal lysine residue giving rise to a $Y_{1}^{\prime \prime}$ ion at $m / z 147$.

\section{Two-Stage CID of a Peptide Mixture}

A limitation in performing pseudo-MS ${ }^{3}$ analyses using an ESI triple quadrupole is the lack of precursor ion selection before the first stage of CID. Thus, the experiments described in this study in general should be confined to pure samples. However, exceptions to this rule have been demonstrated. For instance, selective detection of sphingomyelin in unprocessed total lipid extracts has been demonstrated by pseudo-MS ${ }^{3}$ [21], utilizing the formation of demethylated negative ions by sCID followed by precursor ion scanning for a specific fragment of demethylated sphingomyelin anions. In peptide sequence analysis by pseudo-MS ${ }^{3}$ a 

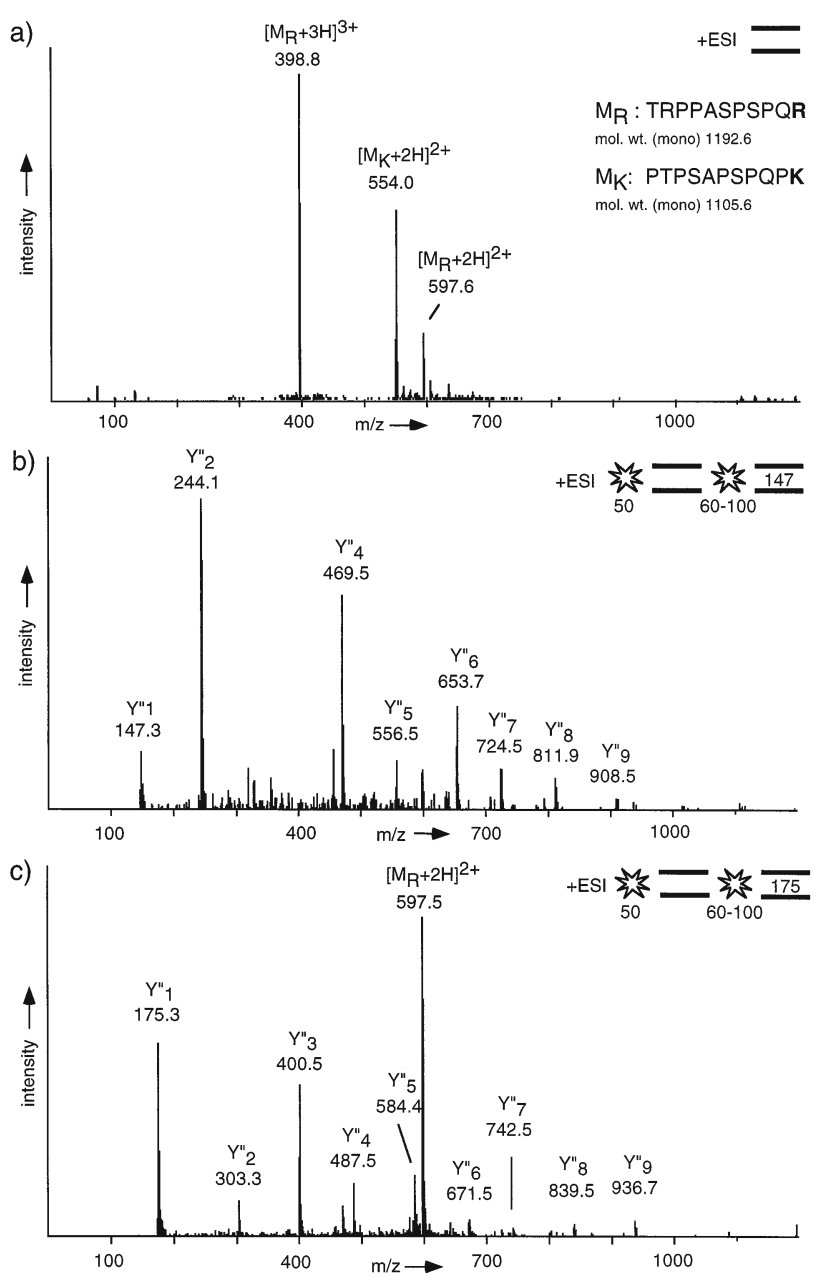

Figure 6. Positive ion nanoESI spectra of a peptide mixture containing the peptide TRPPASPSPQR, "R-peptide" (see Figure 2), plus the peptide PTPSAPSPQPK, "K peptide"; (a) normal ESI spectrum, (b) sCID/precursor of 147 scan ( $\mathrm{Y}_{1}^{\prime \prime}$ ion of $\mathrm{K}$ ) with a sCID offset of $-50 \mathrm{~V}$ and a cCID offset range from -60 to $-100 \mathrm{~V}$, (c) sCID/precursor of 175 scan ( $Y_{1}^{\prime \prime}$ ion of $\mathrm{R}$ ) using the same offset conditions as in (b). The sCID/precursor scans show selectively the $Y^{\prime \prime}$-ion series of the $\mathrm{K}$ peptide and of the $\mathrm{R}$ peptide, respectively.

peptide with a unique structural feature can be sequenced when present in a mixture. A simple example for such an analysis is given in Figure 6 for a two component mixture. Figure 6a gives the normal positive ion ESI spectrum of this sample and the structures of the two peptides, showing that they exhibit different C-terminal residues, namely arginine and lysine. Figures $6 \mathrm{~b}$ and $6 c$ demonstrate that the $Y_{1}^{\prime \prime}$ ion series of these two peptides can be detected separately. As expected, the SCID/precursor of 147 scan displayed in Figure $6 \mathrm{~b}$ predominantly shows the $\mathrm{Y}^{\prime \prime}$ ions of the " $\mathrm{K}$ peptide" abbreviated $M_{\mathrm{K}}$ and accordingly the sCID/ precursor of 175 scan in Figure 6c selectively shows the $\mathrm{Y}^{\prime \prime}$-ion series of the "R peptide" abbreviated $M_{\mathrm{R}}$. Thus it is demonstrated that single fragment ion series can be generated from a peptide present in a mixture, pro- vided that the analyte exhibits a unique and suitable structural element.

\section{Conclusion}

NanoESI performed on a triple quadrupole instrument can be used for recording of peptide fragment ion spectra by a pseudo-MS ${ }^{3}$ experiment involving two stages of collisional activation. Compared to a $\mathrm{MS}^{2}$ analysis an increased level of specificity is achieved that can be used for a selective detection of single fragment ion series with a common structural feature. For instance, selective $Y^{\prime \prime}$-series fragment spectra can be generated from tryptic peptides using a sCID/precursor of $Y_{1}^{\prime \prime}$ scan, because all $Y^{\prime \prime}$ ions in general form a $Y_{1}^{\prime \prime}$ ion in the second stage of collisional activation.

These pseudo-MS ${ }^{3}$ scan modes overcome many difficulties experienced in the interpretation of peptide product ion spectra. Sequence information is easily extracted from single series fragment spectra and the direction of the sequence is also obtained, because the type of fragment ion is determined by the applied scan mode. The specificity of pseudo-MS ${ }^{3}$ also should resolve ambiguities arising from nominal mass overlap of fragment ions. Occasionally occurring peptide backbone rearrangements during fragmentation (see [22]) interfere with this approach, because the interpretation is based on the assumption that a first generation fragment ion has the same primary structure as its precursor molecular ion. Pseudo-MS ${ }^{3}$ scans in a triple quadrupole also have some limitations with respect to sample purity, because the sCID step interacts with a non-mass-selected ion beam. However, this principle does not result in a confinement of pseudo-MS ${ }^{3}$ analyses to pure samples, as has been demonstrated by analysis of peptide mixtures (this study) or unprocessed lipid extracts of cellular origin [21]. Thus, pseudo-MS ${ }^{3}$ scans represent analytically useful extensions of the standard $\mathrm{MS}^{2}$ capabilities of a triple quadrupole.

\section{Acknowledgments}

The author is indebted to A. Tholey and R. Pipkorn for the peptide samples.

\section{References}

1. Biemann, K. Methods Enzymol. 1990, 193, 455-479.

2. Hunt, D. F.; Bone, W. M.; Shabanowitz, J.; Rhodes, J.; Ballard, J. M. Anal. Chem. 1981, 53, 1704-1706.

3. Hunt, D. F.; Yates, J. R., III; Shabanowitz, J.; Winston, S.; Hauer, C. R. Proc. Natl. Acad. Sci. USA 1986, 83, 6233-6237.

4. Roepstorff, P.; Fohlman, J. Biomed. Mass Spectrom. 1984, 11, 601.

5. Stults, J. T.; Lai, J.; McCune, S.; Wetzel, R. Anal. Chem. 1993, 65, 1703-1708.

6. Aeserud, D. J.; Little, D. P.; O'Connor, P. B.; McLafferty, F. W. Rapid Commun. Mass Spectrom. 1995, 9, 871-876.

7. Scoble, H. A.; Martin, S. A. Methods Enzymol. 1990, 193, 519-536. 
8. Takao, T.; Hori, H.; Okamoto, K.; Harada, A.; Kamachi, M.; Shimonishi, Y. Rapid Commun. Mass Spectrom. 1991, 5, 312-315.

9. Schnölzer, M.; Jedrzejewski, P.; Lehmann, W. D. Electrophoresis 1996, 17, 945-953.

10. Schey, K. L.; Schwartz, J. C.; Cooks, R. G. Rapid Commun. Mass Spectrom. 1989, 3, 305-309.

11. Louris, J. N.; Wright, L. G.; Cooks, R. G.; Schoen, A. E. Anal. Chem. 1985, 57, 2918-2924.

12. Schwartz, J. C.; Schey, K. L.; Cooks, R. G. Int. J. Mass Spectrom. Ion Processes 1990, 101, 1-20.

13. Louris, J. N.; Brodbelt-Lustig, J. S.; Cooks, R. G.; Glish, G. L.; vanBerkel, G. J.; McLuckey, S. A. Int. J. Mass Spectrom. Ion Processes 1990, 96, 117-137.

14. Schwartz, J. C.; Jardine, I. Methods Enzymol. 1996, 270, 552-586.
15. Cody, R. B.; Amster, I. J.; McLafferty, F. W. Proc. Natl. Acad. Sci. USA 1985, 82, 6367-6370.

16. Solouki, T.; Pasatolic, L.; Jackson, G. S.; Guan, S. G.; Marshal, A. G. Anal. Chem. 1996, 68, 3718-3725.

17. Schwartz, J. C.; Wade, A. P.; Enke, C. G.; Cooks, R. G. Anal. Chem. 1990, 62, 1809-1818.

18. Lehmann, W. D. J. Am. Soc. Mass Spectrom. 1997, 8, 756-759.

19. Nelson, N. C.; Taylor, S. S. J. Biol. Chem. 1981, 256, 3743-3750.

20. Jedrzejewski, P.; Lehmann, W. D. Anal. Chem. 1997, 69, 294301.

21. Brügger, B.; Erben, G.; Sandhoff, R.; Wieland, F. T.; Lehmann, W. D. Proc. Natl. Acad. Sci. USA 1997, 94, 2339-2344.

22. Tang, X. J.; Thibault, P.; Boyd, R. K. Anal. Chem. 1993, 65, $2824-2834$. 